\title{
Algunos elementos claves del conocimiento especializado del profesor de matemáticas para la gestión de las relaciones área-perímetro
}

\author{
Some key elements of the mathematics teacher's specialised \\ knowledge for the management of area-perimeter relationships
}

\section{Hugo Cesar Cayo Maturana ${ }^{1}$ \\ Luis Carlos Contreras González²}

\begin{abstract}
Resumen: En este trabajo se presentan algunos elementos claves del conocimiento especializado del profesor de matemáticas que consideramos podrían favorecer la gestión del maestro durante el proceso de enseñanza de las relaciones área-perímetro en cursos de Educación Primaria. La información se ha obtenido de dos maestras noveles de Primaria, a través de la observación de las filmaciones de sus clases, las que se desarrollaron en cursos de quinto año de Educación Primaria, de su transcripción y de las entrevistas posteriores. Dicha información ha sido analizada con el modelo analítico Conocimiento Especializado del Profesor de Matemáticas (MTSK), centrándonos solo en aquellos conocimientos que hemos considerado relevantes. Obteniendo finalmente como resultado, en su mayoría, elementos del MTSK correspondientes al Conocimiento Didáctico del Contenido (PCK), ${ }_{1}^{4}$ proponiendo, como elementos claves
\end{abstract}

Fecha de recepción: 14 de septiembre de 2019. Fecha de aceptación: 6 de mayo de 2020.

1 Departamento de Educación, Universidad de Antofagasta, Chile, hugo.cayo@uantof.cl, orcid.org/00000002-9549-8088.

2 Departamento de Didácticas Integradas, Facultad de Educación, Psicología y CC del Deporte, Universidad de Huelva, España, Icarlos@uhu.es, http://orcid.org/0000-0002-0044-2365.

3 MTSK es el acrónimo de Mathematics Teacher's Specialised Knowledge (Conocimiento Especializado del Profesor de Matemáticas).

4 Es el acrónimo acuñado por Shulman que, en castellano, se suele traducir como Conocimiento Didáctico del Contenido y corresponde a uno de los dominios del modelo MTSK. 
del conocimiento especializado, algunas estrategias de enseñanza, ejemplos y recursos didácticos, que consideramos podrían favorecer la gestión de la enseñanza de las relaciones área-perímetro en estudiantes de Primaria.

Palabras claves: Conocimiento especializado, relación área-perímetro, primaria, obstáculos, estrategias.

\begin{abstract}
This study considers several key aspects of the mathematics teacher's specialised knowledge which we believe can benefit the teacher's management of learning in a primary class studying the relationship between area and perimeter. The study focuses on the practices of two new entrants to primary education working in the fifth year, and is based on data drawn from video recordings of their lessons with the corresponding transcriptions, along with follow-up interviews. Analysis was restricted specifically to those areas of knowledge considered relevant, and was carried out within the framework of the Mathematics Teacher's Specialised Knowledge (MTSK) model. The results identify key elements of MTSK corresponding to the domain of Pedagogical Content Knowledge (PCK). These elements form part of the teachers' specialised knowledge, and are given shape in different teaching strategies, examples and educational resources which enhance the primary students' learning of the relationships between area and perimeter.
\end{abstract}

Keywords: Specialised knowledge, relationship between area and perimeter, primary, obstacles, strategies.

\title{
INTRODUCCIÓN
}

Como algunos autores han señalado, la investigación en educación matemática debe tener entre sus prioridades los problemas que derivan de los procesos de enseñanza-aprendizaje en el nivel escolar, abordando las dificultades y los obstáculos que enfrentan los estudiantes (Rico, 2012). De acuerdo con esto, algunos de los temas que deberían ser abordados son la operatoria en el conjunto de los números racionales, la clasificación de figuras geométricas, las fracciones, y las potencias y sus propiedades, identificados como complejos por Pochulu (2009) en estudiantes de último año de Educación Secundaria, o la 
relación entre área y perímetro y el cálculo de área, identificado como problemático en estudiantes para maestros por Montserrat, Boqué y Pañellas (2016).

Si bien son varios los temas que generan dificultades al momento de abordar su enseñanza y aprendizaje, el Seminario de Investigación en Didáctica de la Matemática de la Universidad de Huelva (en adelante SIDM), en cuyo seno se realiza este trabajo, ha declarado explícitamente su interés por profundizar en el estudio de los conocimientos especializados que requiere el profesor al abordar problemas clásicos de enseñanza y aprendizaje de nociones matemáticas, como la relación área-perímetro, la idea de infinito, la clasificación de figuras planas, entre otros (Sosa, Contreras, Gómez, Flores y Montes, 2017). De todos estos, según D'Amore y Fandiño (2007), los problemas de aprendizaje de los conceptos de área y perímetro pueden considerarse como los primeros en haber sido estudiados, como testimonio de esto presentan el famoso problema de las plazas de Galileo: Un pueblo tiene dos plazas A y B; el perímetro de la plaza A es mayor que el perímetro de la plaza B; ¿cuál de las dos plazas tiene el área mayor?

El hecho de que las investigaciones sobre los problemas de aprendizaje de los conceptos de área y perímetro sean antiguas, no implica que sea un tema resuelto. Estos mismos autores, plantearon a un grupo de 43 maestros el problema propuesto por Galileo, y 40 contestaron que la plaza de mayor área era la A (D'Amore y Fandiño, 2007), visualizando en la mayoría de los maestros la misma relación que Stavy y Tirosh (2001) (citados por D'Amore y Fandiño, 2007) observaron en un grupo de estudiantes de distintas edades, que mayor perímetro implica mayor área.

Lo anteriormente expuesto justifica la necesidad de abordar el estudio del conocimiento especializado del profesor concerniente a los procesos de enseñanza de la relación área-perímetro.

\section{ANTECEDENTES}

En los últimos años distintos autores han realizado investigaciones en esta línea; así, Gómez y Vásquez (2015) o Martínez y Pardo (2017) han demostrado que existe confusión entre los conceptos de área y perímetro en estudiantes de Primaria. Gómez y Vásquez (2015) plantean que esta confusión posiblemente se genera en la transición de las intuiciones que traen los estudiantes y los conceptos dados en las salas de clase, mientras que Martínez y Pardo 
(2017), han evidenciado que, al abordar estos conceptos, el trabajo que se realiza en general es de memorización y aplicación de las fórmulas. Esta apreciación es corroborada por García y Carrillo (2006) que señalan que los estudiantes se limitan a la aplicación de fórmulas, en muchos casos de forma indebida y carente de significado.

La creencia de que igualdad de área implica igualdad de perímetro, y viceversa, ha sido estudiada por varios investigadores. Popoca y Acuña (2011) consideran que esta creencia se debe a que los estudiantes tienen más arraigado el pensamiento numérico que el geométrico, lo que les lleva a pensar que si tienen la misma área, tendrán igual perímetro (D’Amore y Fandiño, 2007). Otros estudios señalan que, en algunos casos, predomina el especto bidimensional sobre el unidimensional, lo que lleva a los estudiantes a creer que la figura con el área más grande será la que tenga el perímetro mayor; mientras que, en otros casos, prevalece el aspecto unidimensional, el rectángulo que tenga la mayor altura o base, será el de mayor área (Marchett, Medici, Vighi, y Zaccomer, 2005).

Baltar y Comiti (1993) plantean que es necesario superar la creencia de que igualdad de área implica igualdad de perímetro (y viceversa), antes de abordar las propiedades vinculadas a las variaciones de área y/o perímetro en una misma figura. Popoca y Acuña (2011) observaron que, para algunos estudiantes, el área de una figura (por ejemplo, un rectángulo) mantiene una estrecha relación de dependencia con su forma, por lo tanto piensan que, a distintas dimensiones les corresponde, necesariamente, diferente área. Este problema es especialmente delicado cuando aparece en futuros profesores, como en el estudio de Blanco (1996). Este autor planteó a estudiantes para profesor de Primaria la conjetura de un (hipotético) alumno de Primaria, que tras comparar dos rectángulos ( $4 \times 4$ y $6 \times 4$ ), afirmaba que su ejemplo probaba que a mayor perímetro había también mayor área y observó que 29 de sus 52 estudiantes para profesor, consideraron correcto el razonamiento entregado por un alumno.

Marmolejo, Sánchez y Londoño (2017) concluyeron que las tareas que no propician cambios dimensionales en la figura, no permiten el estudio de las propiedades de variación con respecto a la relación área-perímetro. Según Baltar y Comiti (1993), algunas de las actividades que se desarrollan con mayor frecuencia, favorecen la construcción de la creencia de que área y perímetro varían en el mismo sentido. De alguna forma, esto concuerda con la idea de D'Amore y Fandiño (2007), el obstáculo que se opone a la construcción satisfactoria de las relaciones entre perímetro y área no es solamente de naturaleza 
epistemológica, sino que se origina en las distintas decisiones que toma el maestro, y su naturaleza es, por tanto, didáctica.

Nuestro estudio pretende adentrarse en el conocimiento especializado del profesor de matemáticas acerca de estas relaciones, con la intención de desgranar algunos aspectos de este que podrían favorecer una construcción adecuada por parte de los estudiantes.

\section{FUNDAMENTOS TEÓRICOS}

Nuestro trabajo se fundamenta en dos pilares que están relacionados, el conocimiento especializado del profesor de matemáticas y la ejemplificación. Comenzaremos por el primero y explicaremos después cómo el segundo se integra en este.

Shulman (1986) planteó la existencia de un conocimiento exclusivo de los profesores, un conocimiento que les permite tomar decisiones como, qué deben enseñar, cómo representarlo y cómo resolver problemas generados al abordar un determinado contenido.

Los trabajos de Shulman, relativos a este conocimiento específico de los profesores, llegaron a convertirse en referentes básicos en estudios sobre el conocimiento del profesor. Ball, Thames y Phelps (2008) señalaron hace poco más de una década que sus trabajos presentados en los años 1986 y 1987 habían sido citados en más de 1,200 artículos publicados en revistas especializadas, y han sentado las bases para la creación de distintos modelos que permiten analizar el conocimiento de los maestros. Si hoy hiciéramos una incursión en Google Scholar, encontraríamos que esas dos publicaciones han tenido más de 47,000 citas. De sus aportaciones, cabe destacar la existencia de dos dominios en el conocimiento del profesor: el dominio del conocimiento del contenido y el dominio del conocimiento didáctico del contenido (el denominado paradigma perdido).

Ball et al., (2008), tomando como referencia los dominios propuestos por Shulman, presentaron un modelo para analizar y comprender el conocimiento de los profesores en relación con la enseñanza y el aprendizaje de las matemáticas, Ilamado Conocimiento Matemático para la Enseñanza (MKT). ${ }^{5}$ Desde nuestra perspectiva hay dos aportaciones esenciales en este modelo específico para el conocimiento del profesor de matemáticas. En primer lugar, la distinción, dentro del dominio del conocimiento matemático, de los subdominios del

\footnotetext{
5 MKT es el acrónimo inglés de Mathematical Knowledge for Teaching.
} 
conocimiento matemático especializado (a diferencia del conocimiento matemático común) y del conocimiento en el horizonte matemático; por otro lado, en el dominio del conocimiento didáctico del contenido, de dos subdominios que amalgaman el contenido y su enseñanza y aprendizaje, respectivamente. Sin embargo, algunas investigaciones han detectado dificultades analíticas de este modelo (Montes, Contreras y Carrillo, 2013; Carrillo, Contreras y Flores, 2013) a la hora de ubicar un determinado conocimiento en un solo subdominio, dificultades que los propios autores reconocen en algunos de sus trabajos (Ball y Bass, 2002). Para salvar esta situación, el equipo del SIDM presentó un nuevo modelo denominado Conocimiento Especializado del Profesor de Matemáticas (MTSK), en su diseño se tomaron como base algunas de las potencialidades del MKT y de otros modelos que buscan caracterizar el conocimiento de los profesores de matemáticas (Carrillo et al., 2013).

En MTSK se ha adoptado la definición de conocimiento propuesta por Schoenfeld (2010), quien lo define como aquél que se tiene disponible para su uso; desde la perspectiva del conocimiento del profesor es, por tanto, un conocimiento que se aplica y se pone en juego en los procesos de enseñanza y aprendizaje; es personal, contextualizado, integrado y complejo, práctico, dinámico, y parcialmente tácito. Algunas de estas características sustentan la comprensión del profesor que se encierra en el MTSK (Carrillo et al., 2014; Climent, 2005).

En el MTSK se consideran dos dominios de conocimiento heredados de los trabajos presentados por Shulman $(1986,1987)$ y Ball et al. (2008), el conocimiento del contenido de la materia a enseñar (MK), ${ }^{6}$ y el conocimiento didáctico del contenido a enseñar (PCK) (Carrillo et al., 2014). El MK corresponde al conocimiento que tiene el profesor de la matemática como disciplina científica en el contexto escolar, y PCK considera los conocimientos relacionados con el contenido matemático como objeto de enseñanza-aprendizaje (Carrillo et al., 2014). Ambos dominios están compuestos por tres subdominios y para cada uno se dispone de categorías y subcategorías que permiten analizar y comprender el conocimiento que pone en juego el profesor en el aula (Escudero-Domínguez, Joglar, Correa y Reyes, 2016). Estas categorías han ido emergiendo de diversos estudios empíricos relativos a diferentes contenidos matemáticos y niveles educativos. En lo que sigue, nos referiremos a esos estudios, realizados por miembros

6 En el modelo de conocimiento Especializado del Profesor de Matemáticas (MTSK), el dominio MK (de sus siglas en inglés) denota el conocimiento de la matemática. 
de nuestro grupo, para explicar la estructura del modelo, cuya forma esquemática queda representada en la figura 1.

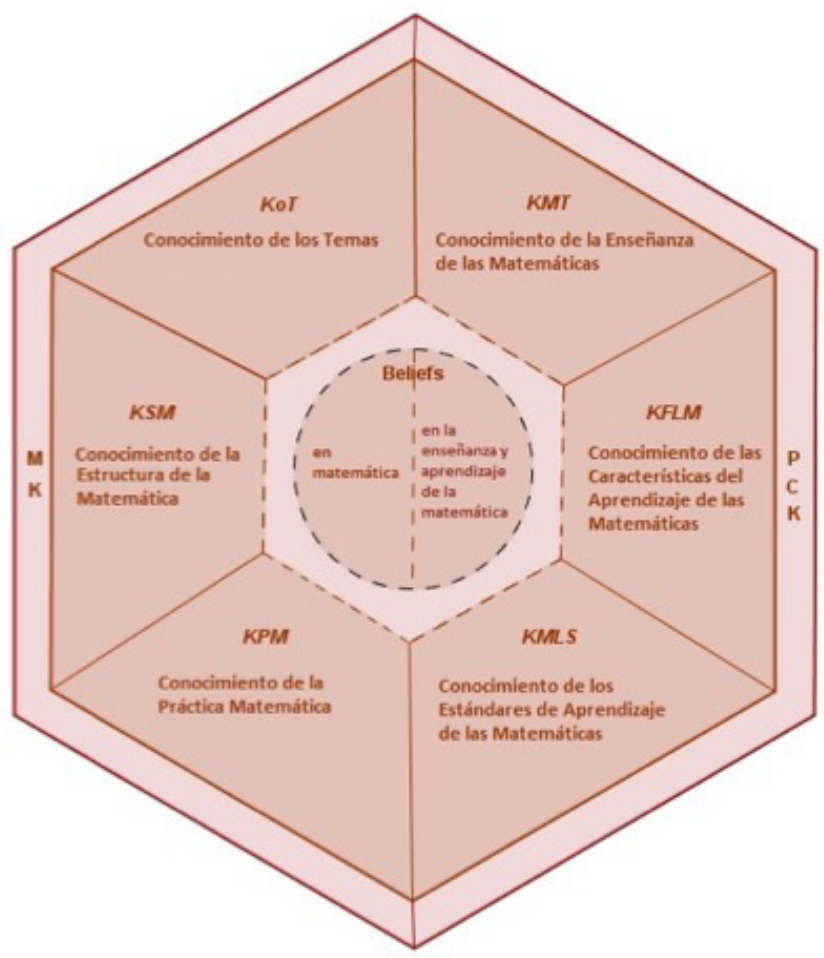

Figura 1. Modelo MTSK (adaptado al castellano de Carrillo et al., 2014).

Los subdominios del MK son el Conocimiento de los Temas (en adelante KoT), ${ }^{7}$ el Conocimiento de la Estructura Matemática (en adelante, KSM), ${ }^{8}$ y el Conocimiento de la Práctica Matemática (en adelante, KPM). ${ }^{9}$

7 KoT es el acrónimo inglés de Conocimiento de los Temas (Knowledge of Topics).

8 KSM es el acrónimo inglés de Conocimiento de la Estructura de la Matemática (Knowledge of the Structure of Mathematics).

9 KPM es el acrónimo inglés de Conocimiento de la Práctica Matemática (Knowledge of Practices in Mathematics). 
Según Liñán, Contreras y Barrera (2016), el KoT considera el conocimiento de las matemáticas en sí mismas, incluye el conocimiento de los conceptos, proposiciones, propiedades, procedimientos o algoritmos, con sus respectivos significados y justificaciones, y se organiza en cuatro categorías: procedimientos; definiciones, propiedades y sus fundamentos; registros de representación; y fenomenología y aplicaciones (Carrillo et al., 2018).

Montes y Climent (2016) establecen que el KSM se define desde una "doble perspectiva", como un conocimiento de conexiones, de cómo se relacionan aquellos elementos que se están considerando en un momento concreto de la enseñanza, con otros; y cómo un conocimiento trasciende la conectividad entre temas. Establecen cuatro categorías: conexiones de complejización, conexiones de simplificación, conexiones auxiliares y conexiones transversales.

El KPM (Flores-Medrano, 2016) es el conocimiento sintáctico; contiene los modos de producción y validación del conocimiento matemático. Se trata de saber cómo se explora y se genera conocimiento en matemática, cómo se establecen relaciones, correspondencias y equivalencias, y cómo se argumenta.

Los subdominios del PCK son el Conocimiento de la Enseñanza de las Matemáticas (en adelante, KMT), ${ }^{10}$ el Conocimiento de las Características del Aprendizaje de las Matemáticas (en adelante, KFLM) ${ }^{11}$ y el Conocimiento de los Estándares de Aprendizaje de las Matemáticas (en adelante, KMLS). ${ }^{12}$

Escudero, Contreras y Vasco (2016) definen el KMT como el conocimiento sobre las características del contenido matemático como objeto de enseñanza; corresponde a lo que el profesor sabe sobre las distintas posibilidades de enseñanza condicionadas por la naturaleza de un determinado contenido. Establecen tres categorías: teorías de enseñanza (personales o institucionales), recursos materiales y virtuales, y estrategias, técnicas, tareas y ejemplos.

En el KFLM se incluye el conocimiento sobre las características inherentes a un contenido matemático, o a la matemática en general, como objeto de aprendizaje, y el conocimiento sobre las características de aprendizajes derivadas de las interacciones de los estudiantes con el contenido (Escudero-Ávila, Climent y Vasco, 2016; Escudero-Ávila, 2015). Se han establecido cuatro categorías: teorías

10 KMT es el acrónimo inglés de Conocimiento de la Enseñanza de las Matemáticas (Knowledge of Mathematics Teaching).

11 KFLM es el acrónimo inglés de Conocimiento de las Características de Aprendizaje de las Matemáticas (Knowledge of Features of Learning Mathematics).

12 KMLS es el acrónico inglés de Conocimiento de los Estándares de Aprendizaje de las Matemáticas (Knowledge of Mathematics Learning Standards). 
sobre aprendizaje (personales o institucionales); fortalezas y debilidades; formas de interacción con el contenido matemático; e intereses y expectativas (Carrillo et al., 2018).

Dentro del KMLS se incluyen los conocimientos sobre qué debería aprender un estudiante en un determinado momento de su escolarización, tomando como referencia el currículo y cualquier otro referente nacional o internacional que desarrolle investigaciones en esta línea. Establecen tres categorías: expectativas de aprendizaje; nivel de desarrollo conceptual o procedimental esperado; y secuenciación con temas anteriores o posteriores (Escudero-Domínguez y Carrillo, 2016).

Los trabajos antes citados nos han mostrado cómo MTSK permite disgregar el conocimiento con propósitos analíticos, para la comprensión de su organización en la mente de un profesor. Se utilizará en este trabajo para analizar y comprender el conocimiento especializado puesto en juego por las profesoras del estudio. Pero, además, como se ha señalado, dentro del subdominio del conocimiento de la enseñanza de las matemáticas se incluye la selección y uso de ejemplos, por ello nos parece adecuado mostrar brevemente nuestro posicionamiento teórico al respecto.

Suffian y Abdul Rahman (2010) consideran que los ejemplos siempre han desempeñado un papel central en la enseñanza de las matemáticas y su uso, por parte de los profesores, ayudan a comprender cómo explican y facilitan la comprensión matemática durante el proceso de enseñanza y aprendizaje. En el mismo sentido se expresan Watson y Mason (2002), quienes afirman que desde hace mucho tiempo se reconoce que las personas aprenden matemáticas principalmente a través de los ejemplos, ya que a través de ellos las definiciones adquieren sentido, y llaman nuestra atención sobre la idea de variación, mostrando su utilidad ya que, al acceder a una amplia variedad de ejemplos, los estudiantes podrán obtener una idea general de lo que están aprendiendo; de acuerdo con estos autores mientras más amplia sea la gama de ejemplos, mayores serán las posibilidades de generalización y conexión. En este sentido, Dienes (2001) (citado por Goldenberg y Mason, 2008) afirmó que los estudiantes necesitan al menos tres ejemplos para tener una idea de un concepto.

Suffian y Abdul Rahman (2010) han observado que seleccionar el ejemplo apropiado es una tarea desafiante que estará determinada en gran medida por el conocimiento especializado que posea el profesor, dicha elección afectará el proceso de aprendizaje de las matemáticas en el aula, por tal razón el profesor debe tener gran cuidado al seleccionar y utilizar un determinado ejemplo. 
La selección y uso de ejemplos será una lente complementaria a través de la cual intentaremos acceder al conocimiento de las profesoras de nuestro estudio.

\section{METODOLOGÍA}

Como hemos señalado, el objetivo de esta investigación es encontrar elementos del conocimiento especializado que puedan considerarse claves para favorecer la construcción de las relaciones área-perímetro en estudiantes de Primaria. Esta investigación se enmarca en el paradigma interpretativo, busca generar conocimiento que favorezca la gestión, por parte del profesor, del proceso de enseñanza-aprendizaje de las relaciones área-perímetro, a partir de las acciones observadas en el aula (Carbajosa, 2011 y Vain, 2012). Corresponde a un tipo de estudio de caso; es una investigación empírica que estudia un suceso, el desarrollo de las clases, en su contexto, abordándolo de manera profunda, con el propósito de obtener un mayor aprendizaje (Durán, 2012), presentando una descripción, una explicación y una valoración de las evidencias, indicios y oportunidades encontradas en los casos seleccionados (Ceballos, 2009); se corresponde, por tanto, con un estudio de caso múltiple, de carácter instrumental (Durán, 2012) en el que se analizaron las observaciones de dos clases de las que se seleccionaron episodios relevantes (López, 2013) para el objetivo de nuestro estudio.

Las informantes son dos maestras noveles, Ely y Ana, se seleccionaron porque ambas debían abordar el mismo objetivo de aprendizaje "diseñar y construir diferentes rectángulos, dados el perímetro o el área o ambos y sacar conclusiones" (MINEDUC, 2013, pp. 92) correspondiente al curriculum establecido para quinto de Primaria y, se mostraron dispuestas a participar en este trabajo. Las dos maestras se encuentran en su primer año de ejercicio docente, han obtenido el título de Profesora de Educación Básica con Mención en Matemática y han egresado de las únicas universidades de la ciudad de Antofagasta que pertenecen al Consejo de Rectores de las Universidades Chilenas. Ely egresó de la Universidad de Antofagasta y se desempeña como maestra en un establecimiento privado; Ana egresó de la Universidad Católica del Norte y se desempeña en un establecimiento municipal.

Para llevar a cabo el análisis de las sesiones se realizó una transcripción literal de las filmaciones de las clases, con el propósito de identificar aquellos episodios que nos permitiesen visualizar evidencias e indicios de los conocimientos 
movilizados por las maestras, vinculados con la enseñanza de la relación área-perímetro. Tomando como base los indicios, se elaboró una entrevista semiestructurada con la cual se buscaba, en la medida de lo posible, transformar los estos en evidencias de los conocimientos movilizados por las maestras.

La revisión de las transcripciones y entrevistas se realizó de forma lineal, a cada episodio que nos entregaba información relevante, se le asignaba uno o unos subdominios del MTSK y un tópico, entendiendo como tópico, el concepto matemático central, que se está abordando en el episodio. Para facilitar el análisis realizamos una primera agrupación por subdominios de aquellos episodios que nos entregaban información, tanto de la transcripción como de la entrevista, por cada maestra. Luego se realizó una agrupación por tópicos.

Es probable que algunos de los elementos que hemos considerado claves del conocimiento especializado para la gestión de la enseñanza de las relaciones área-perímetro ya formen parte de la literatura, pero consideramos que el observar la práctica docente con MTSK activa en el investigador conocimientos que permiten comprender mejor algunos de los obstáculos o dificultades que se pueden presentar al abordar esta temática, estos nuevos conocimientos vienen evocados exclusivamente por la práctica docente (Liñan, 2017).

Con el propósito de centrar la atención en aquellos conocimientos que hemos considerado claves y no en las maestras, al momento de presentar la discusión y conclusiones, nos centraremos en estos conocimientos, apoyándonos en las evidencias obtenidas de los ejemplos utilizados por las dos maestras.

\section{RESULTADOS}

Dado que se identificó un número elevado de episodios ${ }^{13}$ en los cuales se observó la movilización de conocimientos que podríamos llamar "triviales" (como, por ejemplo, "la profesora conoce la diferencia entre área y perímetro"), nos centraremos en aquellos que nos permiten analizar conocimientos que consideramos claves al momento de abordar la relación área-perímetro.

13 El código del episodio $n, X, Y$ indica el episodio n-ésimo de la profesora $X$ (inicial de Ely o Ana) a través de $Y$ (observación de clase (C) o entrevista (E)). 


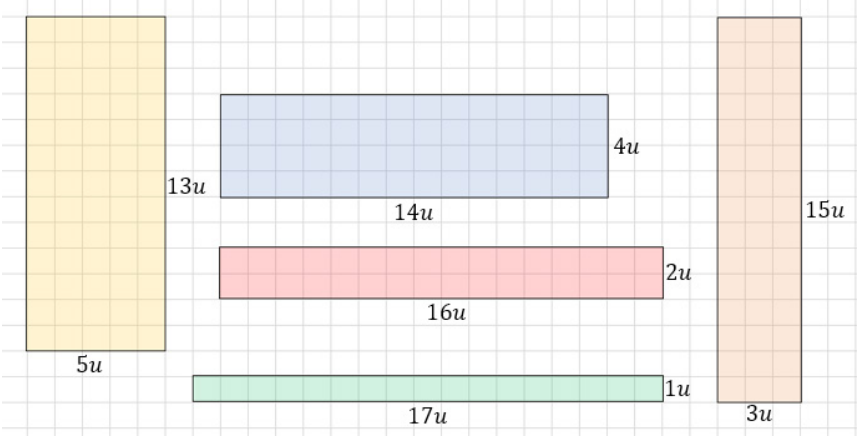

Figura 2. Rectángulos propuestos por la profesora para que los estudiantes los midan con un trozo de hilo.

Ely: $\quad \quad \quad \quad \quad$ a , miren acá, ustedes toman la pita [se refiere a un trozo de hilo] y van a medir el contorno de esta figura, después con la misma medición que tienen de la pita, van a medir los siguientes rectángulos (ver figura 2).

(Episodio 1.E.C.)

Ely: $\quad Y a$, dice: con los cuadrados de cartulina que tienes, armar diferentes rectángulos con áreas iguales, por ejemplo, área 36. Chicos yo solamente les voy a dar el área que sería 36, ustedes tienen que buscar el número [las medidas de los lados]....

(Episodio 2.E.C.)

Ely: $\quad Y a$ de la fila de allá, va a hacer el primero, va a pintar cuatro cuadraditos y nueve, aquí en la pizarra. Pedro ven para acá, a hacer el segundo, Felipe ve a hacer el otro. Pamela anda, tu dijiste que tenías uno distinto (ver figura 3).

(Episodio 3.E.C.)

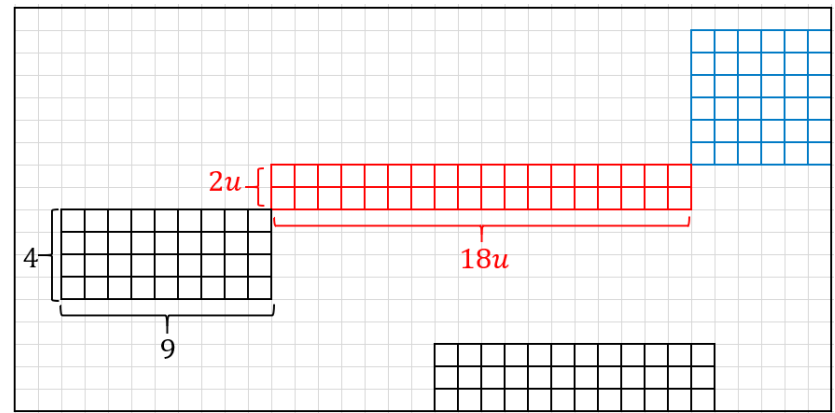

Figura 3. Presentación de los rectángulos construidos por los estudiantes. 
En estos episodios podemos observar evidencias del subdominio de conocimiento para la enseñanza de las matemáticas (KMT) de la maestra; en el primero ha presentado a sus alumnos cinco rectángulos en el pizarrón, todos con diferentes medidas, pero con igual perímetro, los estudiantes dibujan estos rectángulos en su cuaderno, miden el perímetro de uno de ellos con un trozo de hilo, y con la medida obtenida miden el perímetro de los otros rectángulos. Esta actividad nos da evidencias de que la maestra conoce la potencialidad del recurso "hilo" para ofrecer el valor tangible de un perímetro dado, como un valor global más que como suma de longitudes, que permite a los estudiantes comprobar que diferentes rectángulos pueden tener igual perímetro. En el episodio 2.E.C la maestra le pide a los estudiantes que trabajen con cuadraditos, como unidad de medida, y que construyan con ellos rectángulos que tengan un área de $36 \mathrm{u}^{2}$. Podemos ver que la maestra conoce la potencialidad de otro recurso que permite a los estudiantes visualizar que podemos tener diferentes rectángulos con el mismo número de cuadraditos (la misma medida de su área). En el episodio 3.E.C la maestra da evidencias de que conoce la potencialidad de un recurso, la cuadricula, que permite a los estudiantes visualizar las unidades totales del área del rectángulo que se está representando en ella. Todas estas evidencias corresponden a la categoría recursos de subdominio KMT.

Los siguientes episodios corresponden a extractos de la entrevista realizada a la profesora Ely.

Invest: $\quad$ El área aumenta si el perímetro aumenta ¿Está bien la conclusión que obtuvieron los estudiantes?

Ely: $\quad$ No, no sé en qué estaba pensando, a ver, Luis dijo que el área aumenta si el perímetro aumenta, $\mathrm{mmm}$ sí, en realidad sí.

(Episodio 4.E.E.)

Invest: ¿A qué diferencia te refieres?

Ely: $\quad$ A que la figura cambiaba, si yo le ponía más cuadritos igual iba a cambiar la figura, iba a ser diferente el área, si le ponía más cuadritos para igualar el perímetro el área sería diferente, eso quería que entendieran.

(Episodio 5.E.E.)

Invest: ¿ ¿Por qué consideras importante trabajar área y perímetro de rectángulos y cuadrado por separado? 
Ely: $\quad$ Ah, porque son diferentes figuras, unas son iguales, las otras son diferentes. Entonces tienen como otra mecánica, por decirlo así, otra fórmula.

(Episodio 6.E.E.)

En estos episodios se pueden observar evidencias del conocimiento de los temas (KoT) que posee la maestra. En el episodio 4.E.E se observa que la maestra considera la existencia de una relación de dependencia entre área y perímetro, da por correcta la conclusión obtenida por el estudiante, el área aumenta si el perímetro aumenta. En el episodio 5.E.E. la actividad sobre la cual está reflexionando la maestra, consistía en transformar un rectángulo de perímetro 26 en uno de perímetro 36. Se puede observar que la maestra moviliza dos conocimientos con respecto a la relación área-perímetro. Primero, considera que la única forma de aumentar el perímetro de un rectángulo es aumentando su área, en este caso agregando cuadrados a la figura. Segundo, cree que, si cambiamos una de las medidas, área o perímetro, se alterará la otra. Y en el episodio 6.E.E. se observa que domina una clasificación excluyente de los cuadriláteros, no considera al cuadrado como un caso particular de rectángulo.

Los siguientes episodios corresponden a evidencias del MTSK movilizado por la maestra Ana.

Ana: $\quad Y$ acá nos detenemos un rato, menciono lo que ustedes estaban diciendo, según ustedes el rectángulo era más grande que el cuadrado, ¿no?, me dijeron que la base era mayor... (interrumpen los estudiantes).

Alumnos (todos): No, no, son lo mismo.

Felipe: $\quad$ El nueve más el nueve y el uno más el uno da veinte, y el cinco más cinco más cinco más cinco da veinte.

(Episodio 1.A.C.)

Ana:

Ahora, si el perímetro en el rectángulo y en el cuadrado tienen exactamente la misma medida ¿Cómo creen que serían las áreas? ¿Serían iguales? Considerando que tienen el mismo perímetro.

Alumnos (todos): No

(Episodio 2.A.C.)

Ana:

Hasta el momento ¿Cuál es la coincidencia entre los tres rectángulos? 
Pamela: $\quad$ Que tienen la misma área

And:

Ya, los tres rectángulos tienen la misma área ¿Qué va a pasar con el perímetro?

(Episodio 3.A.C.)

Estos tres episodios dan evidencias de su conocimiento sobre la enseñanza de las matemáticas (KMT), en relación con algunas de las estrategias utilizadas por la maestra al abordar la enseñanza de la relación área-perímetro. En el episodio 1.A.C. los estudiantes están observando dos rectángulos con las medidas de sus lados, concluyen que el mayor es aquel que tiene la medida de su base más grande, pero después de realizar el cálculo de los perímetros se percatan de que los rectángulos son isoperimétricos. Esto nos da evidencias de que la maestra conoce una estrategia para hacer reflexionar a los estudiantes sobre la idea errónea de que mayor base implica mayor perímetro. Los episodios 2.A.C. y 3.A.C. nos dan evidencia de que la maestra sabe cómo obtener información del pensamiento de los estudiantes, se puede apreciar cómo propone actividades para comprobar si los estudiantes sostienen ideas incorrectas con respecto a la relación área-perímetro. En el episodio 2.A.C. busca comprobar si los estudiantes sostienen la idea de que igual perímetro implica igual área y en el 3.A.C. si sostienen la idea de que igual área implica igual perímetro. En ambos casos, son evidencias de conocimiento de la categoría estrategia, técnicas, tareas y ejemplos del KMT.

A continuación, se presentan dos episodios más que nos dan evidencias sobre los subdominios de conocimiento de la enseñanza de las matemáticas (KMT) y conocimiento sobre las características del aprendizaje de las matemáticas (KFLM), del MTSK movilizado por Ana; el primero corresponde a su clase y el segundo a su entrevista.

Ana: Hasta el momento ¿Cuál es la coincidencia entre los tres rectángulos? (ver Figura 4)

(Episodio 4.A.C.) 


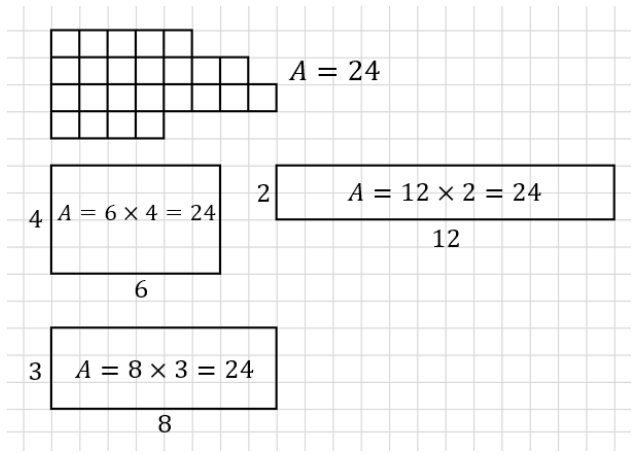

Figura 4. Actividad desarrollada sobre la proyección de una cuadricula.

Ana: $\quad$ El uso de la cuadricula ayuda a visualizar cómo se puede generar un área demarcando una figura. Además de analizar el concepto de área y finalmente comprobando si la manera de calcular el área coincide con la de los cuadrados que están dentro de la figura dibujada.

(Episodio 5.A.E.)

En estos episodios podemos observar más evidencias del KMT movilizado por Ana; vemos que conoce la potencialidad del recurso cuadricula, que permite a los estudiantes visualizar la magnitud del área de los rectángulos representados en ella.

El siguiente episodio corresponde a un extracto de la entrevista que se le realizó a la profesora Ana.

Ana: $\quad$ Sí, quería que se fijaran en la base, seguro que pensarían que al tener una mayor base tendrían mayor área.

(Episodio 6.A.E.)

Este episodio nos da evidencia que sabe que los estudiantes tienden a pensar que el rectángulo que tenga la mayor base será el que tenga la mayor área, que forma parte de la categoría fortalezas y debilidades del KFLM de Ana. 
Los episodios que se presentan a continuación describen aquellas situaciones que se generaron en el aula y que evocaron en el investigador conocimientos vinculados con la enseñanza de la relación área-perímetro.

El primer episodio evocó en el investigador, conocimiento correspondiente a la categoría recursos del subdominio KMT:

Ely: $\quad$ Sí está bien, y cuando mediste con la pita, Katy, te diste cuenta de que todas median igual.

Katy: $\quad$ Similar.

Ely: $\quad$ Ya similar, cierto, porque no va a ser exacto.

(Episodio 7.E.C.)

Es necesario conocer las potencialidades, limitaciones y/u obstáculos que pueden presentar los recursos didácticos seleccionados (KMT, recursos materiales y virtuales), de esta forma los maestros podrán prever los errores que pueden cometer los estudiantes.

En este episodio también se describe una situación que evocó en el investigador conocimiento correspondiente al subdominio KMT:
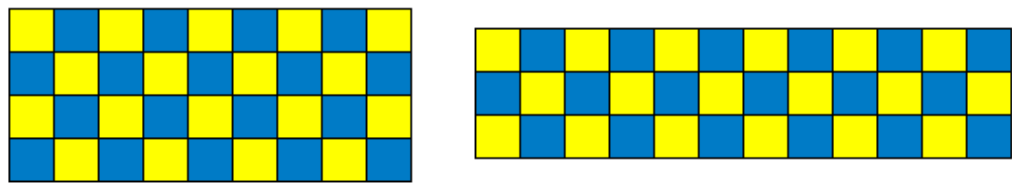

Figura 5. Rectángulos construidos por María y Carla respectivamente.
María: $\quad$ Hay $36(4 \times 9)$.
Ely: $\quad Y$ tú, lo hiciste de.
Carla: $\quad 36(3 \times 12)$.
Ely: $\quad$ ¿Y cuál es la diferencia entre ustedes dos?
Carla: $\quad$ Que ese es más ancho, el mío es más largo y el de ella más ancho.
María: $\quad$ Que yo tengo 4 filas y ella tiene 3.
Ely: $\quad$ ¿Y cuál es más grande?

Carla: $\quad$ Ninguno, porque ambos tienen el mismo número de cuadritos.

(Episodio 8.E.C.) 
Es necesario que los maestros conozcan las oportunidades que pueden ofrecer los distintos recursos didácticos (KMT, recursos materiales y virtuales). La actividad propuesta consistía en que cada estudiante debía construir un rectángulo, utilizando 36 cuadraditos. Durante la clase, cada estudiante trabajó con su rectángulo, pero, al encontrarse estas alumnas con dos rectángulos, ambos construidos con la misma cantidad de cuadrados, pudieron concluir que tenían la misma área, independiente de las medidas.

Los siguientes episodios también evocaron KMT, pero la categoría estrategia, técnicas, tareas y ejemplos:

Ely: $\quad$ ¿Y si te digo perímetro 36, te va a cambiar la figura?

Carlos: $\quad$ No, porque ya me da, si lo sumo todo eso me da 36.

Ely: $\quad$ ¿Seguro? ¿Con los mismos números que me dijiste?

Carlos: $\quad$ Sí, porque nueve por cuatro. Ah no, no, no, porque sí tendría que cambiar la figura. Tendría que hacerla más ancha o más alta, porque ahora lo hice y me dio 26, tengo que agregarle diez más.

Ely: $\quad$ ¿Y qué pasa con el área? Aumenta o disminuye.

Carlos: $\quad$ Aumenta.

(Episodio 9.E.C.)

Pedro: $\quad$ Eso, así ..., si la profe te da el perímetro de 36, tienes que hacer otra figura, por qué, porque ese perímetro está mal, porque si el perímetro es 36 y a ti te dio 26, tienes que cambiar la figura para que el perímetro te dé 36.

Ely: $\quad$ No, es que yo quiero que vean la diferencia ¿iven la diferencia?

Luis: $\quad$ Disminuye.

Ely: $\quad$ Ya Luis dice que disminuye, entonces el área es mucho más grande, si yo voy subiendo, subiendo de número ¿el área se hace más grande?

Luis: $\quad$ Sí.

Ely: $\quad$ Sí, ¿esa es tu conclusión?

Luis: $\quad$ Es como una regla, el área va a aumentar sí o sí.

Ely: $\quad$ El área aumenta si el perímetro aumenta. 
Estos episodios corresponden a la interacción que se generó entre la maestra y los estudiantes cuando realizaron la construcción del rectángulo, utilizando 36 cuadrados. En ellos podemos observar que es necesario que los maestros conozcan cómo gestionar el potencial y las limitaciones de las distintas actividades que desarrollan durante la clase (KMT, estrategias, técnicas, tareas y ejemplos). La actividad tenía como objetivo que los estudiantes comprendieran que rectángulos de igual área no tienen necesariamente igual perímetro, pero al realizar el análisis de un solo rectángulo, no podían observar esta propiedad. También se puede visualizar la necesidad de que los profesores conozcan la pertinencia de las distintas actividades que desarrollan al momento de abordar un determinado contenido (KMT, estrategias, técnicas, tareas y ejemplos); una cosa es estudiar cómo se comportan el área y el perímetro al generar cambios en una misma figura, y otra es analizar qué sucede con el perímetro de figuras que tienen igual área o viceversa.

Este último episodio describe una situación que evocó en el investigador conocimiento de la práctica matemática (correspondiente al subdominio KPM).

Darío: $\quad$ Profe, ¿̇sabe por qué no tienen que ser iguales? Mire, el ancho en una es ocho el largo también. Entonces, la pita no se puede acostumbrar a eso, pero la dirección que nos da es de los números, los números si los sumamos nos dan 36 todos, pero la forma es diferente.

Fabiola: El perímetro es igual pero la forma no.

Darío: $\quad$ Si unos son muy chicos, o nos puede medir muy alto, nos puede faltar o nos puede sobrar, pero los números nos van a dar lo mismo.

(Episodio 11.E.C)

En este episodio se presentan algunas de las conclusiones obtenidas por los estudiantes después de demarcar el perímetro de los cinco rectángulos con un trozo de hilo. Es preciso utilizar las características necesarias para definir el concepto de perímetro (KPM, condiciones necesarias y suficientes para generar definiciones) para no generar obstáculos en el aprendizaje de los estudiantes. Durante la clase se entregaron las siguientes definiciones de perímetro: el contorno; lo de afuera; y suma de los lados, sin hacer referencia al perímetro como una medida longitudinal. Al calcular los perímetros, en todos los rectángulos obtuvieron 36u, pero al medirlos con el hilo no lograron utilizar un único trozo para demarcar los perímetros de todos los rectángulos. Esto llevó a algunos 
estudiantes a realizar una disociación entre la medida del perímetro representada por el hilo y el resultado obtenido al sumar la medida de los lados.

\section{DISCUSIÓN Y CONCLUSIONES}

A continuación, presentamos algunas de las ideas que consideramos podrían favorecer la gestión del profesor durante el proceso de enseñanza de la relación área-perímetro, las cuales se obtuvieron al analizar el MTSK movilizado y evocado por las maestras. Con ello pretendemos poner de relieve nuestra respuesta a la pregunta de investigación: ¿qué elementos claves podemos identificar en el tratamiento de las relaciones área-perímetro? Cada uno de estos conocimientos pertenece a un subdominio específico de MTSK, eso nos permite agrupar los conocimientos en función de sus características, contribuyendo, con ellos, a enriquecer el mapa de conocimientos útiles para gestionar la situación.

Según D'Amore y Fandiño (2007), se ha demostrado ampliamente que estudiantes de todas las edades están convencidos de que igualdad de perímetro implica igualdad de área. Esto ocurre porque los estudiantes tienen más arraigado el pensamiento numérico que el geométrico, estableciendo argumentos intuitivos, por ejemplo, si dos figuras tienen igual perímetro deben tener igual área (Popoca y Acuña, 2011). Es importante que los maestros tengan conocimiento de los errores que comúnmente cometen los estudiantes al abordar una determinada temática (KFLM, fortalezas y debilidades), y que conozcan estrategias que les permitan extraer información del pensamiento de los estudiantes (KMT, estrategias, técnicas, tareas y ejemplos), de esta forma podrán verificar si sostienen o no ideas erróneas. 


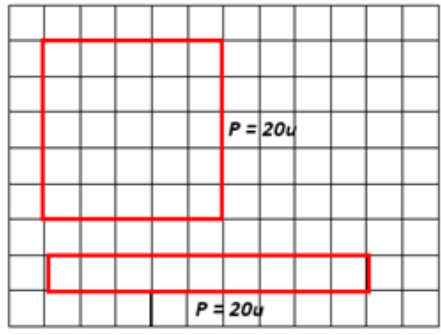

Figura 6. Rectángulos utilizados por la profesora Ana para conocer las concepciones de sus estudiantes.

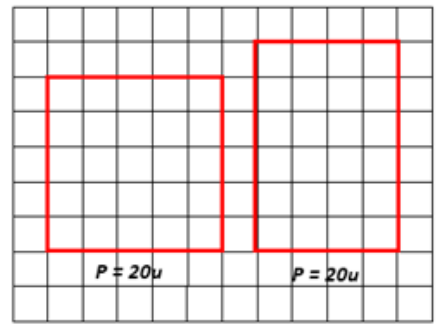

Figura 7. Rectángulos que proponemos para extraer información de las concepciones de los estudiantes.

Así, una estrategia para extraer información del pensamiento de los estudiantes podría ser preguntarles qué piensan que pasaría con el área de dos figuras que ya sabemos que tienen igual perímetro ¿̇serán iguales o diferentes? Pero debemos tener cuidado con el ejemplo que seleccionamos para realizar el análisis. La profesora Ana desarrolló la actividad con dos rectángulos, uno de 5x5 y otro de 1x9 (Figura 6), los de mayor y menor área que se pueden obtener con perímetro 20u y trabajando con números enteros. Al preguntar a los estudiantes qué pasaría con el área de estos rectángulos que tienen igual perímetro, respondieron que eran diferentes. Visualmente era evidente que las áreas no medían lo mismo, situación que podría haber impedido que los estudiantes expusieran sus verdaderas creencias. Creemos que sería pertinente utilizar rectángulos que tengan áreas con medidas similares (Figura 7). Esto podría motivar a los estudiantes a exteriorizar sus verdaderas creencias.

La clase de Ely nos permitió observar que, un trozo de hilo es un recurso que se debe considerar al momento de abordar el hecho de que diferentes rectángulos pueden tener igual perímetro, pues tiene la potencialidad de permitir a los estudiantes comprobar la existencia de esta propiedad sin necesidad de obtener 
el valor del perímetro mediante suma de lados (KMT, recursos materiales y virtuales). Si entregamos a los estudiantes un conjunto de rectángulos de igual perímetro y un trozo de hilo con longitud igual al perímetro de los rectángulos, podrían verificar de forma concreta que todos los perímetros tienen la misma longitud (KMT, estrategias, técnicas, tareas y ejemplos), a pesar de que algunos se vean más largos o más anchos. Si bien podrían ver que los perímetros son iguales con el simple hecho de sumar las medidas de los lados, Marmolejo y Vega (2012) plantean que el aprendizaje de la geometría ocurre mediante la coordinación de actividades de visualización, razonamiento y construcción, como sugiere esta situación.

Con respecto al uso del trozo de hilo, es necesario que los maestros conozcan un obstáculo que se podría presentar. Algunos estudiantes pueden tener dificultades al momento de manipular el trozo de hilo, esto les impediría visualizar que todos los rectángulos son isoperimétricos (KMT, recursos materiales y virtuales). Esta situación podría provocar un obstáculo didáctico, llevando a los estudiantes a realizar una disociación entre la medida del perímetro representada por el trozo de hilo y el resultado obtenido al sumar las medidas de los lados, situación que se evidenció en la clase de Ely. Creemos que los maestros deben ser conscientes de esta limitación (KFLM, fortalezas y dificultades). Consideremos que por un lado han comprobado o podrán comprobar mediante la suma de las medidas de los lados que todos lo rectángulos tienen igual perímetro, pero al momento de demarcarlos no todos tienen la misma longitud.

El que algunos estudiantes hayan realizado una disociación nos dio evidencias de la importancia que tiene que los profesores conozcan cuáles son las condiciones necesarias y suficientes para establecer definiciones apropiadas (KPM). Durante la clase se presentaron distintas definiciones para perímetro, pero ninguna hacía referencia al perímetro como medida longitudinal o a su unidad de medida; el haber presentado una definición que incorpora estas características, podría haber provocado que los estudiantes cuestionaran la disociación que estaban realizando. Ripoll (2001, p. 53) propone algunas estrategias para facilitar el aprendizaje de las matemáticas, la primera consiste en "utilizar en el lenguaje habitual del aula un vocabulario matemático que frecuentemente no se utiliza o que se sustituye por términos no precisos desde el punto de vista de la Matemática".

Para sacar un mayor provecho de esta actividad, es necesario que los maestros conozcan las oportunidades de enseñanza que nos pueden ofrecer los distintos ejemplos, al momento de asignarle el valor al perímetro de los rectángulos (KMT, 
estrategias, técnicas, tareas y ejemplos). Consideramos pertinente asignarle al perímetro un valor que posibilite la construcción del cuadrado y que nos permita presentar un número amplio de rectángulos; de esta forma no solo podremos trabajar la propiedad de que distintos rectángulos pueden tener igual perímetro, también podríamos abordar la propiedad de que entre los rectángulos de igual perímetro el de mayor área es el cuadrado- ver Figura 8 (KoT, propiedades y sus fundamentos). Si bien Ely trabajó con rectángulos de perímetro 36u, no consideró el cuadrado de lados 9u (Figura 2). De acuerdo con lo declarado por la maestra en la entrevista, cuadrado y rectángulos son figuras diferentes (KoT, definiciones propiedades y sus fundamentos) probablemente la elección del ejemplo realizada por la maestra estuvo determinada por su nivel de conocimiento de la clasificación de los cuadriláteros (Suffian y Abdul Rahman, 2010). En nuestra opinión sostener esta clasificación excluyente de los cuadriláteros no le permitió abordar con esta u otra actividad la propiedad de que, entre los rectángulos de igual perímetro el de mayor área es el cuadrado (KMT, estrategias, técnicas, tareas y ejemplos).
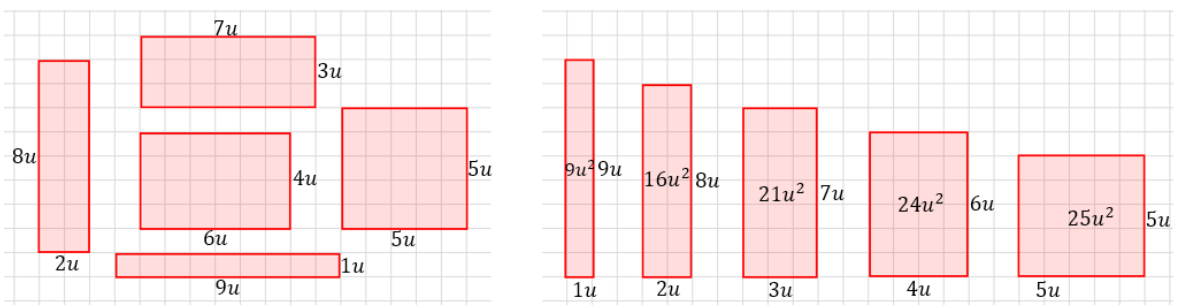

Figura 8. Ejemplo de rectángulos propuestos para potenciar la actividad.

Marchett et al., (2005) concluyeron que, en algunas ocasiones, al momento de comparar las áreas prevalece el aspecto unidimensional de la figura. Es importante que los profesores sepan que algunos estudiantes tenderán a creer que el rectángulo que tenga la mayor base o la mayor altura tendrá mayor área (KFLM, fortalezas y debilidades). Esta creencia podría obstaculizar que los alumnos comprendan que diferentes rectángulos pueden tener igual área. Hemos observado en nuestro estudio que trabajar con cuadricula (KMT, recursos materiales y virtuales) puede favorecer la gestión del proceso de enseñanza al momento de abordar esta temática. Mántica, Gotte y Dal Maso (2006) han evidenciado que, si los estudiantes no cuentan con papel cuadriculado, presentan 
una dificultad mayor al momento de construir figuras de un área determinada, y según lo declarado por la profesora Ana, una de las ventajas que ofrece la cuadricula es que permite a los estudiantes comprobar si la cantidad de cuadrados que están dentro de la figura coinciden con el resultado obtenido al calcular el área. Esto permite a los estudiantes verificar el resultado obtenido al calcular el área de dos o más figuras, en caso de que no "creyeran" que diferentes rectángulos pueden tener igual área. Como ya hemos mencionado, en geometría el aprendizaje se produce mediante la coordinación de actividades de construcción, visualización y razonamiento (Marmolejo y Vega, 2012), idea que de alguna forma respalda el uso de la cuadricula.

Otro recurso que nos permitiría abordar esta creencia son los cuadrados de cartulinas para utilízalos como unidad de medida de área (KMT, recursos materiales y virtuales). Antes de trabajar con ellos es indispensable que los maestros conozcan las potencialidades y limitaciones que les puede ofrecer una actividad desarrollada con este recurso. Si se pide a cada estudiante que construya un rectángulo utilizando un determinado número de cuadrados, esta actividad no permitiría que visualicen que podemos tener diferentes rectángulos con igual área, situación que se observó en la clase de Ely, donde la mayoría de los estudiantes centró su atención en la construcción y análisis de su rectángulo. Pero, si los estudiantes trabajan en grupos y se le pide a cada integrante que construya un rectángulo, utilizando un determinado número de cuadrados, con la condición de que todos deben construir rectángulos diferentes, al momento de realizar el análisis podrán observar varios ejemplos, de esta forma aumentará la probabilidad de que puedan obtener una generalización de lo que están aprendiendo (Watson y Mason, 2002); podrán concluir que, independiente de las medidas del rectángulo, todos tienen la misma área ya que todos utilizaron el mismo número de cuadrados para su construcción. Esta situación se pudo observar en el aula de Ely, en cuyo caso, al acercarse la maestra a dos alumnas que compartían mesa y preguntarles ¿̇uál de las dos había construido un rectángulo más grande? (haciendo referencia al área), ambas respondieron que eran iguales porque trabajaron con la misma cantidad de cuadrados.

Mántica, Gotte y Dal Maso (2006) plantean que el hecho de que dos figuras tengan la misma área induce a los estudiantes a creer que tendrán igual perímetro. Es importante que los profesores conozcan estas creencias que podrían tener algunos estudiantes (KFLM, fortalezas y dificultades). Si los estudiantes ya han interiorizado que todos los rectángulos construidos tienen la misma área, bastaría con desarrollar una actividad que permitiera analizar los perímetros de 
los rectángulos para que visualizase que igualdad de área no implica igualdad de perímetro (KMT, estrategias, técnicas, tareas y ejemplos).

Consideramos que trabajar con un área de 36 cuadrados sería apropiado, ya que nos permitiría construir 5 rectángulos diferentes $1 \times 36$; 2x18; 3x12; 4 ×8 y 6x6, incluyendo un cuadrado, siguiendo la idea de Dienes (2001) (citado por Goldenberg y Mason, 2008: 186) en relación con el número de ejemplos que necesitan los estudiantes para tener una idea de un concepto. Otros números que se podrían considerar para establecer la medida del área, el 60, permite un rectángulo más, pero no permite la construcción del cuadrado; los números 49 y 81 permiten la construcción del cuadrado, pero solo se pueden construir 2 y 3 rectángulos respectivamente; el 100 permite la construcción del cuadrado y también, se pueden hacer 5 rectángulos diferentes, pero debemos considerar que trabajar con muchos cuadrados como unidad de área, puede dificultar el aprendizaje, transformado el recurso en un distractor (KMT, estrategias, técnicas, tareas y ejemplos). El trabajar con los 5 rectángulos que se pueden construir con los 36 cuadrados, nos permite generar la instancia para que los estudiantes visualicen que de todos los rectángulos de igual área, el de menor perímetro será el cuadrado- ver Figura 9 (KoT, definiciones, propiedades y sus fundamentos).

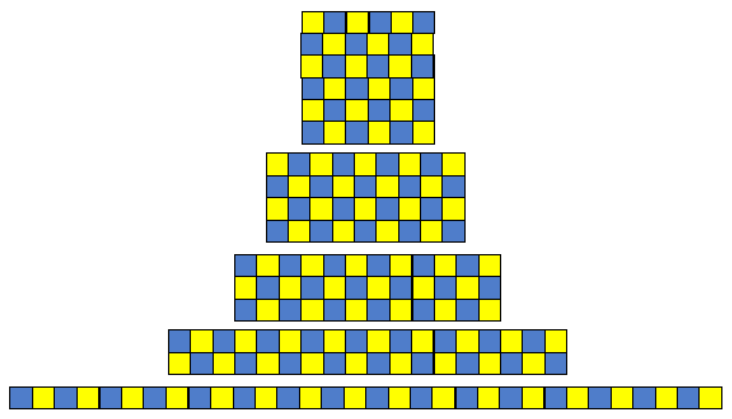

Figura 9. Rectángulos propuestos para potenciar la actividad.

D’Amore y Fandiño (2007) concluyeron que los obstáculos que se oponen a la correcta construcción del conocimiento sobre la relación área-perímetro no son solo de naturaleza epistemológica, sino que son básicamente de naturaleza didáctica. Es necesario que los maestros conozcan la pertinencia de las distintas actividades que realizan. Si bien ninguna de las actividades tenía como propósito central analizar cómo se comportan el área y el perímetro al momento de realizar 
variaciones en alguna de las medidas del rectángulo, durante la clase de Ely se generaron algunas instancias que permitieron a los estudiantes construir conocimiento en relación con esta temática. Esto ocurrió cuando la profesora le pidió a los estudiantes que trasformaran el rectángulo que habían construido con los 36 cuadrados, uno de 9x4, cuyo perímetro era de 26, en uno de perímetro 36. Baltar y Comiti (1993) plantean que, actividades similares a la desarrollada por la maestra, posiblemente dan origen a la idea errónea de que área y perímetro varían de la misma forma, ya que los estudiantes tienden a aumentar el área para lograr que crezca el perímetro. Lo que concuerda con lo observado en la clase de Ely, aumentaron el área para obtener un perímetro de 36u y concluyeron que el área aumenta si el perímetro aumenta, conclusión aceptada por la maestra (KoT, definiciones propiedades y sus fundamentos) y por un gran número de estudiantes para profesor (Blanco, 1996).

Hemos pretendido con nuestro trabajo poner de manifiesto, y al alcance de toda la comunidad educativa, especialmente de formadores de maestros y estudiantes para maestros, aquellos elementos claves que observamos en nuestro estudio de casos y que consideramos que podrían favorecer la gestión del proceso de enseñanza de las relaciones área y perímetro. MTSK se ha mostrado relevante como instrumento de análisis que, nos ha permitido organizar el conocimiento puesto en juego en el aula y discutir acerca de conocimientos que permitiría una gestión alternativa a la analizada. Estamos convencidos que existen otros conocimientos que también serán clave en este proceso, como la literatura ya lo ha demostrado. Una posible continuidad de este estudio, sería realizar esta investigación con profesores expertos, enriqueciendo así el elenco de conocimientos esenciales para una gestión eficaz de los procesos de enseñanza y aprendizaje de las relaciones estudiadas.

\section{AGRADECIMIENTOS}

El Gobierno de España (EDU2013-44047-P y EDU2016-81994-REDT), y el centro de investigación COIDESO (Universidad de Huelva, España) apoyan esta investigación. 


\section{REFERENCIAS}

Ball, D.L. y Bass, H. (2002). Toward a Practice-Based Theory of Mathematical Knowledge for Teaching. En Proceedings of Annual Meeting of Canadian Mathematics Education Study Group (pp. 3-14). CMESG.

Ball, D., Thames, M. H., y Phelps, G. (2008). Conocimiento del contenido para la enseñanza: ¿Qué lo hace especial? Revista de formación docente, 59(5), 389-407.

Baltar, P. y Comiti, C. (1993). Difficultes rencontrees par des élèves de cinquième en ce qui concerne la dissociation aire/perimetre pour des rectangles. Petit, 34, 5-29.

Blanco, L. (1996). Aprender a enseñzar geometría. Una experiencia en la formación del profesorado. Épsilon, 34, 47-58.

Carbajosa, D. (2011). Debate desde paradigmas en la evaluación educativa. Perfiles Educativos, 33(132), 181-190.

Carrillo, J., Climent, N., Montes, M., Contreras, L. C., Flores, E., Escudero, D., ... Muñoz, M. C. (2018). The mathematics teacher's specialised knowledge (MTSK) model. Research in Mathematics Education, 20(3), 236-253.

Carrillo, J., Contreras, L. C., Climent, N., Escudero-Avila, D., Flores-Medrano, E., y Montes, M. A. (2014). Un marco teórico para el conocimiento especializado del profesor de matemáticas. Universidad de Huelva Publicaciones.

Carrillo, J., Contreras, L.C., y Flores, P. (2013). Un modelo de conocimiento especializado del profesor de matemáticas. En L. Rico, M. C. Cañadas, J. Gutiérrez, M. Molina e I. Segovia (Eds.), Investigación en Didáctica de la Matemática. Libro homenaje a Encarnación Castro (pp. 193-200). Comares.

Ceballos-Herrera, F. A. (2009). El informe de investigación con estudio de casos. Magis. Revista Internacional de Investigación en Educación, 1(2), 413-423.

D'Amore, B. y Fandiño, M. (2007). Relaciones entre área y perímetro: convicciones de maestros y de estudiantes. Revista Latinoamericana de Investigación en Matemática Educativa, 10, 39-68.

Durán, M. M. (2012). El estudio de caso en la investigación cualitativa. Revista nacional de administración, 3(1), 121-134.

Escudero-Ávila, D., Contreras, L.C., y Vasco, D. (2016). Conocimiento de la enseñanza de la matemáticas (KMT). En J. Carrillo, L.C. Contreras y M. Montes (Eds.), Reflexionando sobre el conocimiento del profesor. Actas de las II Jornadas del Seminario de Investigación de Didáctica de la Matemática de la Universidad de Huelva (pp. 35-41). SGSE.

Escudero-Ávila, D., Climent, N., y Vasco, D. (2016). Conocimiento de las características del aprendizaje de las matemáticas (KLFM). En J. Carrillo, L.C. Contreras y M. Montes 
(Eds.), Reflexionando sobre el conocimiento del profesor. Actas de las II Jornadas del Seminario de Investigación de Didáctica de la Matemática de la Universidad de Huelva (pp.42-48). SGSE.

Escudero-Domínguez, A., Joglar, N., Corrêa, D., y Reyes, A. (2016). Retrospectiva de las investigaciones sobre conocimiento especializado del profesor de matemáticas. En J. Carrillo, L.C. Contreras y M. Montes (Eds.), Reflexionando sobre el conocimiento del profesor. Actas de las II Jornadas del Seminario de Investigación de Didáctica de la Matemática de la Universidad de Huelva (pp. 69-86). SGSE.

Escudero-Domínguez D. y Carrillo, J. (2016). Conocimiento de los estándares de aprendizaje de las matemáticas (KMLS). En J. Carrillo, L.C. Contreras y M. Montes (Eds.), Reflexionando sobre el conocimiento del profesor. Actas de las II Jornadas del Seminario de Investigación de Didáctica de la Matemática de la Universidad de Huelva (pp. 49-54). SGSE.

Flores-Medrano, E. (2016). Conocimiento de la práctica matemática (KPM). En J. Carrillo, L.C. Contreras y M. Montes (Eds.), Reflexionando sobre el conocimiento del profesor. Actas de las II Jornadas del Seminario de Investigación de Didáctica de la Matemática de la Universidad de Huelva (pp. 30-34). SGSE.

Garcia, G. y Carrillo, J. (2006). Relación entre perímetro y área: el caso de Patricia y las interacciones. Investigación en educación matemática: actas del X Simposio de la Sociedad Española de Investigación en Educación Matemática (pp. 185-194). Instituto de Estudios Altoaragoneses.

Goldenberg, P. y Mason, J. (2008). Arrojando luz sobre y con espacios de ejemplo. Estudios educativos en matemáticas, 69(2), 183-194.

Gómez, T. y Vásquez, K. (2015). Área y perímetro de cuadriláteros en estudiantes colombianos de. En P. Scott, y Á. Ruiz (Ed.), XIV Conferencia Interamericana de Educación Matemática. (pp. 1-9). CIAEM.

Liñan, M. M., Contreras, L.C. y Barrera, V. (2016). Conocimiento de los Temas (KoT). En J. Carrillo, L.C. Contreras y M. Montes (Eds.), Reflexionando sobre el conocimiento del profesor. Actas de las II Jornadas del Seminario de Investigación de Didáctica de la Matemática de la Universidad de Huelva (pp. 12 -20). SGSE.

Liñan, M. M.. (2017). Conocimiento Especializado en Geometría en un aula de 5o de Primaria. (Tesis doctoral no publicada). Universidad de Huelva.

López, W. (2013). El estudio de casos: una vertiente para la investigación educativa. Educere, 17(56), 139-144

Mántica, A., Gotte, M., y Dal Maso, M. (2006). Una propuesta para el tratamiento del concepto de área en EGB. Acta Latinoamericana de Matemática Educativa, 19, 108-114. 
Marchett, P., Medici, D., Vighi, P., y Zaccomer, E. (2005). Comparing perimeters and areas. Childrens preconceptions and spontaneous procedures. En Bosch, M. (Ed.), Proceedings of the 4th Congress of the European Society for Research in Mathematics Education (pp. 766-776). FUNDEMI IQS. Universitat Ramon Llull.

Marmolejo, G. y Vega, M. (2012). La visualización en las figuras geométricas. Importancia y complejidad de su aprendizaje. Educación Matemática, 24(3), 7-32.

Marmolejo, G., Sánchez, N., y Londoño, S. (2017). Conocimiento visual de los educadores al promover el estudio de la relación perímetro-área. Revista Electrónica de Investigación en Educación en Ciencias, 12(2), 18-28.

Martínez, M. y Pardo, S. (2017). Concepciones de estudiantes de educación básica sobre perímetro y área. Eco matemático, 8(1), 71-80.

MINEDUC. (2013). Programa de estudio. Quinto año Básico. Ministerio de Educación.

Montes, M. A., Contreras, L. C. y Carrillo, J. (2013). Conocimiento del profesor de matemáticas: Enfoques del MKT y del MTSK. En A. Berciano, G. Gutiérrez, A. Estepa y N. Climent (Eds.), Investigación en Educación Matemática XVII (pp. 403-410). SEIEM.

Montes, M.A. y Climent, N., (2016). Conocimiento de la estructura matemática (KSM). En J. Carrillo, L.C. Contreras y M. Montes (Eds.), Reflexionando sobre el conocimiento del profesor. Actas de las II Jornadas del Seminario de Investigación de Didáctica de la Matemática de la Universidad de Huelva (pp. 21 -29). SGSE.

Montserrat, D. N., Boqué, M. y Pañellas, M. (2016). Dificultades en conceptos matemáticos básicos de los estudiantes para maestro. International Journal of Developmental and Educational Psychology. Revista INFAD de Psicología. 1(1), 419-430.

Pochulu, M. (2009). Análisis y categorización de errores en el aprendizaje de la matemática en alumnos que ingresan a la universidad. Colección Digital Eudoxus, 8.

Popoca, M. y Acuña, C. (2011). Cambio en figuras de área igual, conservación y relaciones figurales. Acta Latinoamericana de Matemática Educativa, 24, 541-550.

Ripoll, M. (2001). Algunas estrategias para facilitar el aprendizaje de las matemáticas. Números. Revista de didáctica de las matemáticas, 45, 53-60.

Rico, L. (2012). Aproximación a la investigación en Didáctica de la Matemática. Avances de Investigación en Educación Matemática, 1, 39-63.

Schoenfeld, AH (2010). Cómo pensamos: una teoría de la toma de decisiones orientada a objetivos y sus aplicaciones educativas. Routledge.

Shulman, L. (1986). Aquellos que entienden. El crecimiento del conocimiento en la enseñanza. Investigador Educativo, 15(2), 4.14.

Sosa, L., Contreras, L.C., Gómez, I., Flores, E., y Montes, M. (2017). Síntesis, problemas abiertos, preguntas para reflexión. En J. Carrillo y L.C. Contreras (Eds.), Avances, utilidades y retos del modelo MTSK: (pp. 71-79). CGSE. 
Suffian, H., y Abdul Rahman, S. (2010). Teacher's choice and use of examples in the teaching and learning of mathematics in primary school and their relations to teacher's pedagogical content knowledge (PCK). Procedia-Social and Behavioral Sciences, 8(5), 312-316.

Vain, P. D. (2012). El enfoque interpretativo en investigación educativa: algunas consideraciones teórico-metodológicas. Revista de Educación, 4(4), 37-45.

Watson, A. y Mason, J. (2002). Ampliación de espacios de ejemplo como estrategia de aprendizaje / enseñanza en matemáticas. En A. Cockburn, y E. Nardi (Eds.), Actas de la 26므 Conferencia del Grupo Internacional para la Psicología de la Educación Matemática, Volumen 4 (pp. 377-385). Universidad de East Anglia.

Hugo César Cayo Maturana

Dirección: Departamento de Educación, Universidad de Antofagasta, Chile, 02800

Teléfono: $\quad+56975282898,+34644820370$ 